\title{
Preface
}

\section{Intensive Care Units as a Place to Manage Pulmonary Emboli}

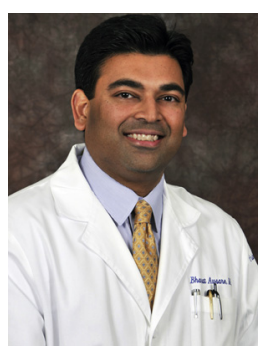

Bharat Awsare, MD

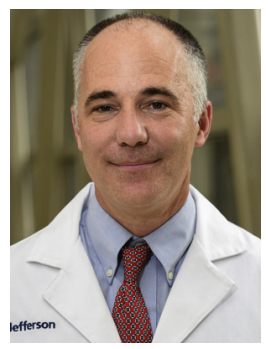

Michael Baram, MD Editors

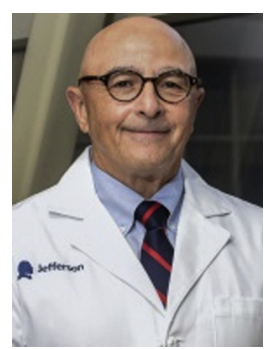

Geno Merli, MD

There was once a time when the main considerations for the acute treatment of pulmonary emboli (PE) were anticoagulation, inferior vena caval interruption, and thrombolytics. The diagnosis, risk-stratification, treatment, and follow-up of PE has become more complicated. Due to the heterogeneity of PE patients (medical, oncology, orthopedics, surgery, neurosurgery, pediatrics, and obstetrics), a variety of alternate options began to develop. The management of PE became more complex due to the expanded role of catheter-based therapies, surgical thrombectomies, and cardiac-assist technologies, such as right ventricular-assist devices and extracorporeal support. Formulating a comprehensive treatment plan now necessitates an interaction between multiple specialties and departments. The intensive care unit (ICU) is designed not only to provide support for the most critically ill but also to facilitate the multidisciplinary care provided by intensivists, hematologists, vascular specialists, cardiologists, interventional radiologists, pharmacists, and cardiothoracic surgeons. When an institution engages the multiple disciplines with PE expertise to develop algorithms of best practice and processes of care, patient care can be improved through optimized management of local resources. The same organized team can develop institutional algorithms as well as provide direct patient care. The most complex and critically ill of the PE patients pose unique challenges to the established management guidelines due to comorbidities, acute organ failure, and physiologic instability. In this issue of Critical Care Clinics, we explore the unique aspects of management of the subset of PE patients requiring ICU care. A special effort was made to include a wide variety of perspectives from many disciplines. The goal of this issue is to help intensivists organize a methodical approach to assess, diagnose, risk-stratify, and treat PE in the ICU. Different articles address treatment algorithms on reperfusion therapies, while others focus on the cardiovascular and pulmonary support of the ICU patient. The 
editors of this issue would like to thank all contributors who have shared their knowledge and time to make this multidisciplinary issue of Critical Care Clinics come to fruition.

\author{
Bharat Awsare, MD \\ Department of Medicine \\ Division of Pulmonary and Critical Care \\ Jefferson University Hospital \\ Korman Lung Institute \\ 834 Walnut Street, Suite 650 \\ Philadelphia, PA 19107, USA \\ Michael Baram, MD \\ Department of Medicine \\ Division of Pulmonary and Critical Care \\ Jefferson University Hospital \\ Korman Lung Institute \\ 834 Walnut Street, Suite 650 \\ Philadelphia, PA 19107, USA \\ Geno Merli, MD \\ Department of Medicine and Surgery \\ Division of Vascular Medicine \\ Jefferson University Hospital \\ 111 South 11th Street, Suite 6210 \\ Philadelphia, PA 19107, USA \\ E-mail addresses: \\ Bharat.Awsare@jefferson.edu (B. Awsare) \\ Michael.Baram@jefferson.edu (M. Baram) \\ Geno.Merli@jefferson.edu (G. Merli)
}

